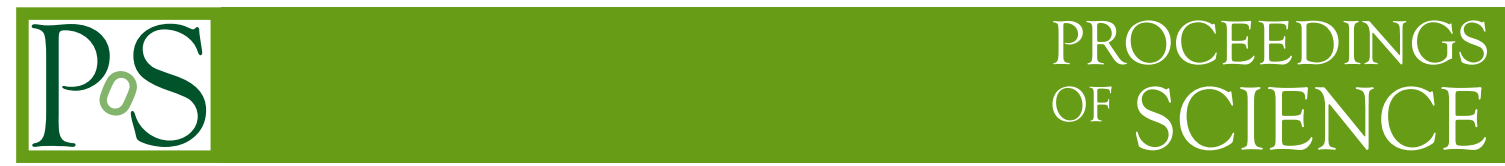

\title{
Last results from the WEBT and GASP
}

\author{
Claudia M. Raiteri* and Massimo Villata \\ INAF, Osservatorio Astronomico di Torino, Italy \\ E-mail: raiteri@oato.inaf.it, villata@oato.inaf.it

\section{for the WEBT collaboration}

Born in 1997, the Whole Earth Blazar Telescope (WEBT) is a network of optical, near-infrared, and radio telescopes that in concert have the capability to obtain continuous, high-temporaldensity monitoring of blazars during time-limited campaigns on specific objects.

The GLAST-AGILE Support Program (GASP) was organized within the WEBT to provide optical-to-radio long-term continuous monitoring of a list of selected $\gamma$-ray-loud blazars during the operation of the AGILE and GLAST satellites.

We present the results of the first GASP effort, targetting the BL Lac object $0716+714$ in September-November 2007, when the source was detected by AGILE. In October an outburst was detected simultaneously in the optical and radio bands.

We also show some results of the last WEBT campaign on the quasar-type blazar 3C 454.3 in the 2007-2008 observing season. In this period 3C 454.3 was very active, showing pronounced optical outbursts, and it was detected by AGILE in July and then in November-December. Moreover, several noticeable episodes of very fast (i.e. intranight) variability were observed in the optical light curves.

Workshop on Blazar Variability across the Electromagnetic Spectrum April 22-25, 2008

Palaiseau, France

\footnotetext{
* Speaker.
} 


\section{Introduction}

The Whole Earth Blazar Telescope (WEBT; http://www.oato.inaf.it/blazars/webt) consortium is an international collaboration among astronomers devoted to blazar studies. It was founded in autumn 1997 by John Mattox, from the Boston University, as a network of optical telescopes whose distribution in longitude on the Earth surface can potentially produce continuous monitoring over the 24 hours per day. In 2000 the WEBT leadership passed to Massimo Villata, from the Torino Observatory, who gave it a Constitution. Soon after, observers in the radio and near-IR bands joined the collaboration, making it become a formidable multifrequency tool for blazar studies.

The WEBT organizes multifrequency campaigns on specific objects, with variable duration in time (from a few weeks to several months), in which more than 100 telescopes have participated so far. Moreover, WEBT projects usually involve observations at high energies, from both satellites and ground-based $\mathrm{TeV}$ telescopes, allowing us to reconstruct the source emission behaviour over a wide range of wavelengths.

In its 10 years of activity, 23 campaigns have been carried out by the WEBT, each one led by a Campaign Manager, who is responsible for defining the observing strategy, for data collection, analysis, and publication. Up to now, the WEBT has produced about 60 publications. The multifrequency light curves obtained from the WEBT campaigns are stored in the WEBT archive and are available upon request to the WEBT President starting from one year after the publication of the results.

In view of the $\gamma$-ray data release by the AGILE and GLAST satellites, the WEBT has started a new project, the GLAST-AGILE Support Program (GASP). This involves those WEBT observers who can provide long-term continuous monitoring for a sample of blazars. The list of observed sources is displayed in Table 1: some of them are observed with higher priority (1).

To promptly discover interesting states of the sources, data reduction, collection and assembling must be fast. Hence, the GASP observers are required to quickly perform data reduction and magnitude calibration according to a common choice of photometric sequences, and send the data to the WEBT President, who checks them and constructs the light curves. The detection of a bright state can trigger ToO observations by the high-energy satellites.

The main aim is to obtain simultaneous observations in the optical and $\gamma$-ray bands to investigate the expected correlation between flux variations in the two bands. This issue was already tackled during the operation of the Compton Gamma Ray Observatory (CGRO) in 1991-2000, but not completely understood.

In this paper we present the results obtained by the GASP on the BL Lac object $0716+714$ at the beginning of its operation (see also [1]). We also show some of the results achieved by the WEBT in the last campaign on the quasar-type blazar 3C 454.3 (see also [2]).

For both the above mentioned sources, a cross-correlation analysis between the GASP-WEBT data and the AGILE data is under way.

\section{The GASP on $0716+714$}

The GASP project officially started in early September 2007, just before a strong $\gamma$-ray detection of $0716+714$ by the AGILE satellite (see D'Ammando et al., these proceedings). Because of 


$\begin{array}{lccccc}\text { IAU Name } & \text { Object Name } & \text { Priority } & \text { RA (J2000.0) } & \text { DEC (J2000.0) } & \text { Redshift } \\ & & & {[\mathrm{h} \mathrm{m} \mathrm{s}]} & {[\mathrm{d} \mathrm{m} \mathrm{s}]} & z \\ 0219+428 & \text { 3C 66A } & 1 & 022239.6 & +430208 & 0.444 \\ 0235+164 & \text { AO 0235+16 } & 1 & 023838.9 & +163659 & 0.94 \\ 0420-014 & \text { PKS 0420-01 } & 2 & 042315.8 & -012033 & 0.914 \\ 0528+134 & \text { PKS 0528+134 } & 2 & 053056.4 & +133155 & 2.06 \\ 0716+714 & \text { S5 0716+71 } & 1 & 072153.4 & +712036 & 0.3 \\ 0735+178 & \text { PKS 0735+17 } & 1 & 073807.4 & +174219 & 0.424 \\ 0827+243 & \text { OJ 248 } & 2 & 083052.1 & +241100 & 0.939 \\ 0829+046 & \text { OJ 49 } & 2 & 083148.9 & +042939 & 0.173683 \\ 0836+710 & \text { 4C 71.07 } & 2 & 084124.3 & +705342 & 2.172 \\ 0851+202 & \text { OJ 287 } & 1 & 085448.9 & +200631 & 0.306 \\ 0954+658 & \text { S4 0954+65 } & 1 & 095847.2 & +653355 & 0.368 \\ 1101+384 & \text { Mkn 421 } & 1 & 110427.3 & +381232 & 0.030021 \\ 1156+295 & \text { 4C 29.45 } & 1 & 115931.8 & +291444 & 0.729 \\ 1219+285 & \text { ON 231 } & 1 & 122131.7 & +281359 & 0.102 \\ 1226+023 & \text { 3C 273 } & 2 & 122906.7 & +020309 & 0.158339 \\ 1253-055 & \text { 3C 279 } & 1 & 125611.1 & -054722 & 0.5362 \\ 1510-089 & \text { PKS 1510-08 } & 2 & 151250.5 & -090600 & 0.36 \\ 1611+343 & \text { DA 406 } & 2 & 161341.0 & +341248 & 1.401 \\ 1633+382 & \text { 4C 38.41 } & 1 & 163515.5 & +380804 & 1.81357 \\ 1641+399 & \text { 3C 345 } & 2 & 164258.8 & +394837 & 0.5928 \\ 1652+398 & \text { Mkn 501 } & 2 & 165352.2 & +394537 & 0.033663 \\ 1739+522 & \text { 4C 51.37 } & 2 & 174037.0 & +521143 & 1.375 \\ 1807+698 & \text { 3C 371 } & 2 & 180650.7 & +694928 & 0.051 \\ 2155-304 & \text { PKS 2155-304 } & 1 & 215852.0 & -301332 & 0.116 \\ 2200+420 & \text { BL Lacertae } & 1 & 220243.3 & +421640 & 0.0686 \\ 2230+114 & \text { CTA 102 } & 2 & 223236.4 & +114351 & 1.037 \\ 2251+158 & \text { 3C 454.3 } & 1 & 225357.7 & +160854 & 0.859 \\ 2344+514 & \text { 1ES 2344+514 } & 2 & 234704.8 & +514218 & 0.044\end{array}$

Table 1: GLAST-AGILE Support Program (GASP) Source List.

this detection and the following optical outburst, also other WEBT members joined the observing effort on this source.

The top panel of Fig. 1 shows the $R$-band data collected by the GASP and WEBT in AugustNovember 2007. The first yellow strip indicates the period of the first AGILE pointing in September 2007, including the strong $\gamma$-ray detection reported by [3]. Since the optical observing season was at the beginning, the optical data are sparse. However, one can see that an optical flare occurred in the same period of the AGILE detection.

After a period of flickering, in mid October the optical flux started to rise, reaching a peak of $R=12.15$. This level is comparable to the brightest states ever observed and triggered intensive observations at all wavelengths, in particular by AGILE (second yellow strip in the figure) and 

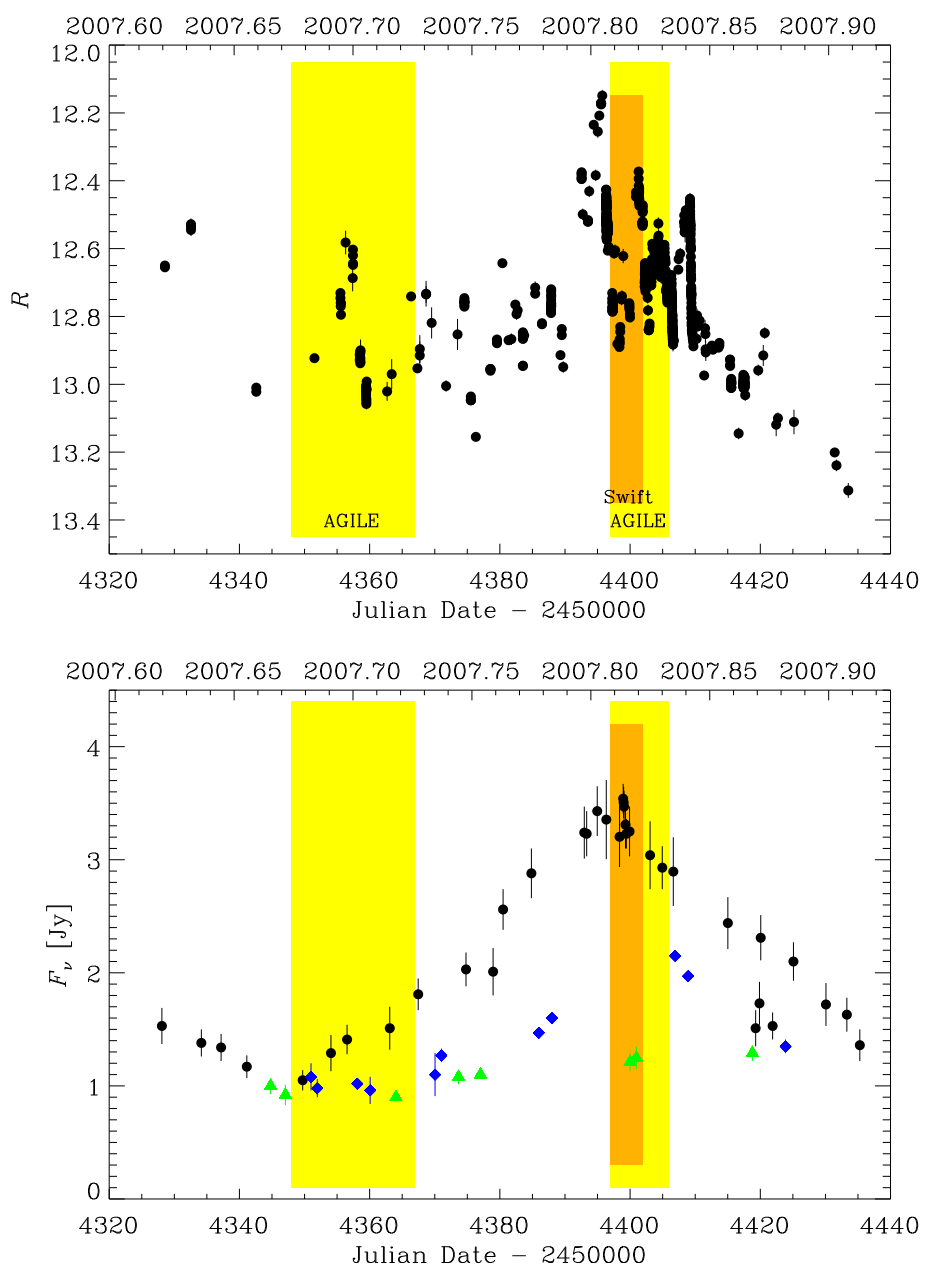

Figure 1: Top: $R$-band light curve of 0716+714 in August-November 2007, composed with data from the GASP and other WEBT telescopes. The yellow strips indicate the two periods of AGILE observations, the orange strip marks the period of ToO observations by Swift. Bottom: Radio light curves at $37 \mathrm{GHz}$ (black circles), 14.5 GHz (blue diamonds), and $8 \mathrm{GHz}$ (green triangles).

Swift ToO pointings (orange strip).

Observations at $37 \mathrm{GHz}$ (see Fig. 1, bottom panel) show that an outburst was occurring also in that radio band. The optical and radio outbursts appear roughly contemporaneous. However, when seen in detail, the two events show a different behaviour, as the radio event is smoother and seems to start before.

A contemporaneous optical-radio event has sometimes been observed in blazars. However, usually radio events are observed to lag behind the optical ones by several weeks or months. In the case of 0716+714, [4] noticed that in the 1994-2001 observing period, major optical outbursts may have modest radio counterparts. Thus, the optically-emitting jet region is sometimes not completely opaque to the high radio frequencies. In particular, it is quite transparent to them in the case of the 2007 outburst. Lower radio frequencies are at least partially absorbed, as the $14.5 \mathrm{GHz}$ delay and the strongly-inverted radio spectrum indicate (see the bottom panel of Fig. 1). 


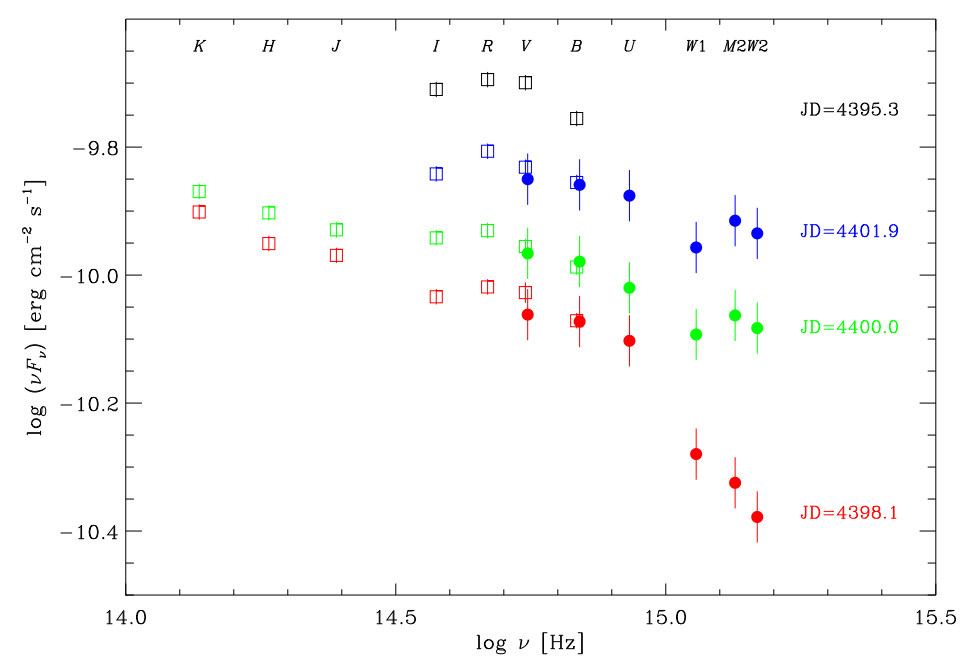

Figure 2: Spectral energy distributions (SEDs) of $0716+714$ in late October 2007, constructed with $V, B, U, W 1, M 2, W 2$ data from Swift-UVOT (filled circles) and ground-based GASP-WEBT $B, V, R, I, J, H, K$ data (empty squares). Different colours refer to different epochs, which are indicated on the right.

Source SEDs built with $V, B, U, W 1, M 2, W 2$ data from Swift-UVOT and $B, V, R, I, J, H, K$ data from GASP-WEBT are displayed in Fig. 2. Various epochs characterized by different brightness levels were selected. Data were corrected for Galactic extinction. The SEDs show two main features: the bluer-when-brighter behaviour (especially in the UV band), which is typical of BL Lac objects, and the wavy shape of the spectra. It remains to be clarified whether this latter feature is due to some physical reason or it is produced by calibration problems and/or uncertainties in the Galactic absorption estimate.

\section{The WEBT on $3 C 454.3$}

The quasar-type blazar 3C 454.3 underwent a phase of high activity in summer and then in autumn-winter 2007-2008, which was intensively monitored in the radio-to-optical bands by the WEBT in the ambit of a long-term campaign on this object ${ }^{1}$. AGILE detected this source first in late July (see [8]), and then in November-December 2007. Observations by Swift were also triggered.

The $R$-band light curve constructed with WEBT data is shown in Fig. 3, where it is possible to see several noticeable outbursts. Moreover, many episodes of fast (i.e. intranight) variability were observed, especially in November-December, involving flux changes of several tenths of mag in a few hours. Some of them are displayed in Fig. 4.

The most striking event was detected on December 12 (marked with a black arrow in Fig. 3), when a flux increase of about 1.1 mag in 1.5 hours was followed by a fall of about 1.2 mag in 1 hour. This is one of the fastest variations ever observed in blazars. We think that it is most likely a

\footnotetext{
${ }^{1}$ The WEBT has been intensively monitoring 3C 454.3 since the occurrence of the exceptional outburst of 2004 2005 (see [5], [6], [7], [2])
} 


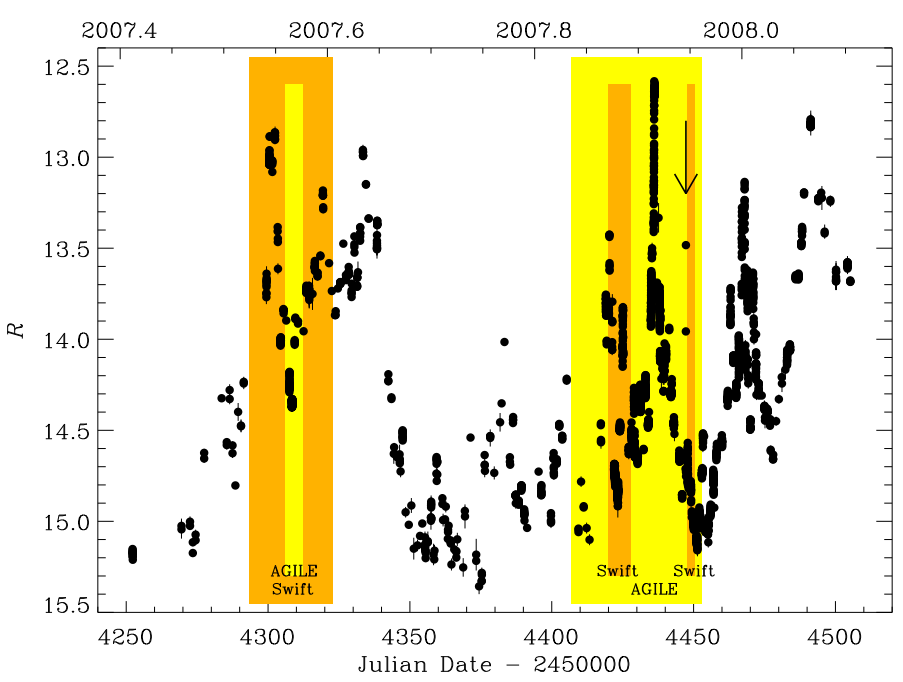

Figure 3: $R$-band light curve of 3C 454.3 in the 2007-2008 observing season constructed with WEBT data. The yellow strips indicate the two periods of AGILE observations, the orange strips highlight the periods of ToO observations by Swift. The black arrow marks the most striking episode of intranight variability occurred on December 12, 2007.
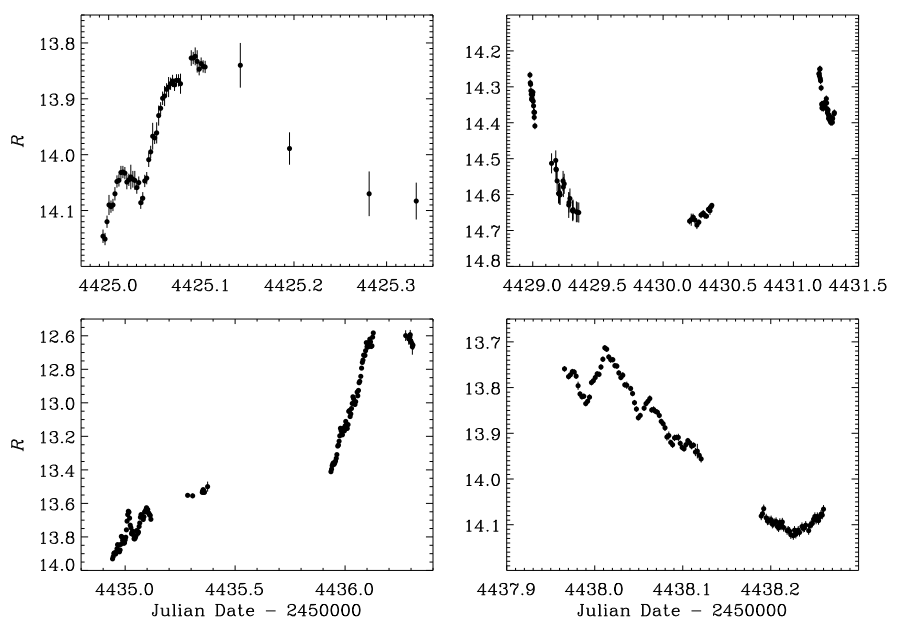

Figure 4: Noticeable episodes of very fast variability in the $R$-band light curve.

real variation in the jet emission. Indeed, alternative explanations, such as a $\gamma$-ray burst (GRB) in the host galaxy, or a microlensing effect, e.g. by a MACHO in our Galaxy, seem more unlikely. The limited sampling prevents us from any consideration on the shape and chromatism of the event.

We constructed broad-band SEDs with contemporaneous data from the WEBT (radio to optical) and Swift-UVOT (optical-UV). All data have been corrected for Galactic extinction, whose amount in the UV is strongly affected by the $2175 \AA$ bump due to dust absorption. Consequently, the shape of the spectrum in the UV band has to be taken with caution. However, a polynomial fit of the SEDs from the radio to the UV data shows that the optical-UV SED becomes more and more concave as the flux decreases, suggesting the presence of a rather stable UV excess. Indeed, a UV excess was found by [7] when analysing XMM-Newton data taken in July and December 


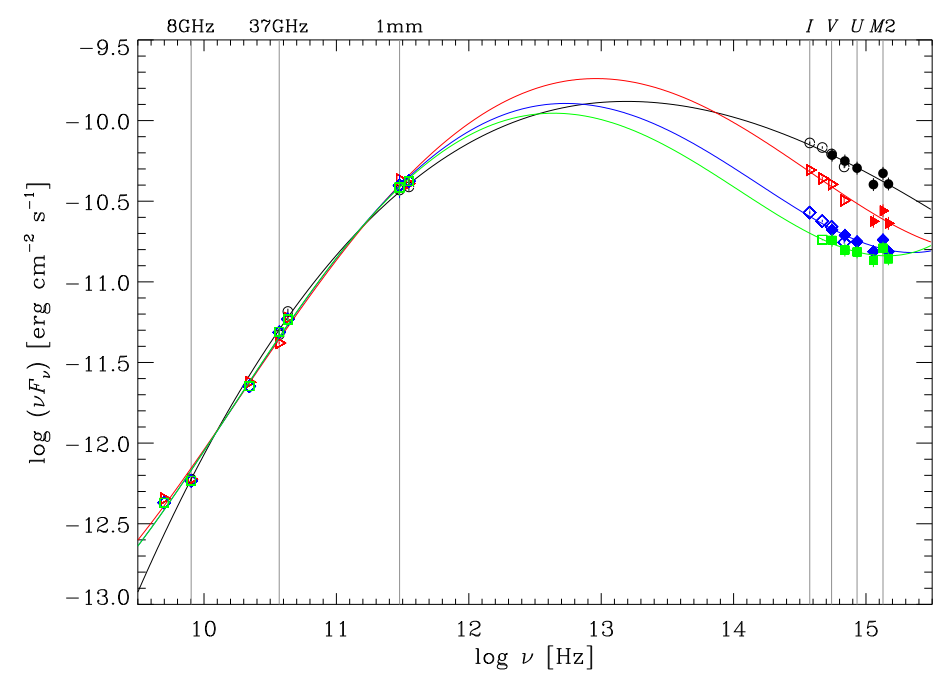

Figure 5: Spectral energy distributions (SEDs) of 3C 454.3 at different brightness levels in NovemberDecember 2007. Contemporaneous data are plotted with the same symbol and colour; filled symbols refer to Swift-UVOT data, while empty symbols represent ground-based observations. The curves show polynomial fits to the radio-to-UV data at the various epochs.

2006, which was interpreted as the contribution from the accretion disc. Our SEDs would confirm the picture according to which the thermal contribution from the disc is overwhelmed by the beamed synchrotron emission when the source is bright, and it can only be recognised when the non-thermal flux is low.

\section{References}

[1] M. Villata et al., 2008, Multifrequency monitoring of the blazar 0716+714 during the GASP-WEBT-AGILE campaign of 2007, A\&A 481 (L79)

[2] C. M. Raiteri et al., 2008, The high activity of 3C 454.3 in autumn 2007. Monitoring by the WEBT during the AGILE detection, $A \& A 485$ (L17)

[3] A. Giuliani et al., 2007 AGILE detection of a gamma-ray source possibly associated with Blazar TXS 0716+714, The Astronomer's Telegram 1221

[4] C. M. Raiteri et al., 2003, Optical and radio behaviour of the BL Lacertae object 0716+714, A\&A $402(151)$

[5] M. Villata et al., 2006, The unprecedented optical outburst of the quasar 3C 454.3. The WEBT campaign of 2004-2005, A\&A 453 (817)

[6] M. Villata et al., 2007, The radio delay of the exceptional 3C 454.3 outburst. Follow-up WEBT observations in 2005-2006, A\&A 464 (L5)

[7] C. M. Raiteri et al., 2007, WEBT and XMM-Newton observations of $3 C 454.3$ during the post-outburst phase. Detection of the little and big blue bumps, A\&A 473 (819)

[8] S. Vercellone et al., 2008, AGILE detection of a strong gamma-ray flare from the blazar 3C 454.3, ApJ 676 (L13) 